\title{
Editorial
}

Stéphane Jaisson and Philippe Gillery

\section{Innovative approaches in diabetes diagnosis and monitoring: less invasive, less expensive... but less, equally or more efficient?}

https://doi.org/10.1515/cclm-2018-0549

Criteria for screening, diagnosing and monitoring diabetes mellitus rely on well-defined protocols, which have been based for decades on venous (for screening/diagnosis) or capillary (for monitoring) glucose determinations. It is now well admitted that $\mathrm{HbA}_{1 \mathrm{c}}$ may also be used in current practice, not only for monitoring but also for diagnosis of diabetes [1]. $\mathrm{HbA}_{1 \mathrm{c}}$ is a typical product of non-enzymatic glycation, a general process that refers to the irreversible binding of simple oses or their by-products to proteins. In particular, $\mathrm{HbA}_{1 \mathrm{c}}$, which results from the binding of glucose to the $\mathrm{N}$-terminal extremities of globin $\beta$ chains, retrospectively reflects the cumulative binding of glucose to hemoglobin depending on glycemia levels during the 4-8 weeks preceding the assay [2]. Although there is still some debate on the respective use of the two biomarkers, these recommendations indicate that both instantaneous glucose concentrations and integrated markers of glycation can be used in these circumstances, both approaches seeming equally efficient in providing valuable clinical information.

However, although these worldwide strategies have been established, the development of new approaches using innovative methods of protein glycation evaluation cannot be disregarded. Two types of reasons justify this research: (i) economical constraints, especially in developing countries, and (ii) implementation of non invasive techniques. In this issue of Clinical Chemistry and Laboratory Medicine, Monteyne et al. [3] describe a technique of near-infrared (NIR) spectroscopy for evaluating glycation in nails meeting the two criteria.

It has been shown for several years, especially using in vitro approaches, that physical methods like infrared (IR) or Raman spectroscopies could be valuable tools for evaluating protein glycation [4-6] or other nonenzymatic posttranslational modifications (NEPTMs) like carbamylation [7]. Spectroscopic methods provide specific spectra reflecting structural characteristics of proteins, especially regarding secondary and tertiary structures, which are altered by NEPTMs-induced changes, especially in the case of glycation [8]. For example, Raman spectroscopy has proved to be able to distinguish between spectra generated by glycated and non glycated forms of both hemoglobin [9] and albumin [4], allowing the development of semiquantitative methods for glycation evaluation. Different applications of IR spectroscopy have also been applied to the detection of glycation-induced changes in proteins. Before the present publication, the same team had evaluated the ability of attenuated total reflection Fourier-transform infrared (ATR-FTIR) spectroscopy to detect glycation on the same materials [10]. However, this approach required that the nail clippings are powdered prior analysis, thus limiting its use in a routine practice. The NIR equipment used in the present publication may be connected to optic fibers allowing the analysis to be directly performed on complete fingernails, which makes this method more convenient and suitable for a routine practice [3].

The use of nails as primary materials is also an interesting approach. It is well known that glycation products, especially advanced glycation end products (AGEs), accumulate in extracellular matrix-rich tissues like skin, and that their evaluation provides an integrative retrospective view of glycemic control on long time periods, depending on the half-life of constituting proteins [11, 12]. As these investigations generally require biopsies, they cannot be carried out in current practice. However, original non-invasive investigations, precisely using spectroscopy, must be mentioned, such as those performed on human lips with a FTIR spectroscopy device [13]. Practically speaking, nails constitute valuable materials because they can be obtained without causing skin damage. Besides, they are physiologically more relevant than other easy-to-obtain samples like hair because they are continuously in close contact with blood vessels and interstitial fluid [14]. Nail protein glycation has been proposed as marker in diabetes more than 30 years ago [15]. However, the application in clinical settings had rarely been proposed [16] until the authors of the commented paper proposed different new 
strategies to assess glycemic control in African countries $[3,14,17]$. All these studies used nail clippings as materials for evaluating glycation, not only by spectroscopic methods but also by assaying fructosamine directly on the nail samples [17]. In the latter case, nail clippings were simply dipped into a fructosamine assay reagent, providing a simple way to measure protein glycation, especially suitable for countries with low economical outcome. The present study has the additional advantage to be performed without needing any reagent.

The paper published in this issue focuses on the application of NIR spectroscopy to the diagnosis of diabetes mellitus, claiming that it could be considered an alternative technique. However, broader applications could be considered because these approaches could also constitute valuable tools in diabetes follow-up. The road has been opened several years ago with the non-invasive measurement of skin autofluorescence as a direct reflect of skin glycation due to AGE deposition [18]. As a matter of fact, this measurement has proven to be a valuable mean to assess patient "metabolic burden", and skin autofluorescence has been shown to be correlated with systemic AGE concentrations and to the development of various diabetes mellitus complications $[19,20]$. The physical spectroscopic methods described here could also represent promising applications for non-invasive investigations.

Thus, this approach and comparable ones are promising and should lead to new developments. However, their use must still be considered preliminary in clinical situations, and there is probably a long way before their use in routine. Indeed, many markers of glycation, especially AGEs, have been proposed in the last decades for providing additional information to established biomarkers in the follow-up of patients with diabetes mellitus [21, 22]. However, because of the lack of method comparison, harmonization or standardization [23], the use of these promising markers remains limited [24]. Comparable remarks apply to this NIR spectroscopy method and other similar ones. Glycation-induced spectral changes have clearly been evidenced, allowing the use of the method in clinical situations, but the subtle molecular changes responsible for spectral alterations have not yet been clearly identified. Therefore, at that step, the method is only qualitative allowing discriminating between diabetic and non-diabetic subjects, but challenges related to the establishment of reliable and transferable quantification procedures have still to be faced. Indeed, the ability of these methods to provide quantitative information is a key factor for reaching the intended clinical purposes. When this goal is reached, calibration processes must be established with suitable materials and procedures, and then harmonized in order to allow between-center comparability of results. Such pitfalls limit the comparisons of results from autofluorescence measurements, which can however reach a satisfactory degree in precision when performed in a single center. Additional aspects have to be considered like the expertise of the operators, which must be strictly assessed and controlled, and the costs of the devices, which must be taken into account in the economical assessment, even if they are eventually paid off.

To conclude, screening, diagnosis and monitoring of diabetes mellitus will probably benefit in the future from the input of new methodological approaches, like the NIR spectroscopic method described here. However, their strengths, relying mainly on their non-invasiveness and their low cost, must be considered in light of their currently limited quantitative properties and the necessary demonstration of their effectiveness for clinical purpose when compared to currently accepted methodologies and protocols. A long way of stimulating experimentations is still to go!

Author contributions: All the authors have accepted responsibility for the entire content of this submitted manuscript and approved submission.

Research funding: None declared.

Employment or leadership: None declared.

Honorarium: None declared.

\section{References}

1. American Diabetes Association. 2. Classification and diagnosis of diabetes: standards of medical care in diabetes-2018. Diabetes Care 2018;41:S13-27.

2. Gillery P. A history of HbA1c through Clinical Chemistry and Laboratory Medicine. Clin Chem Lab Med 2013;51:65-74.

3. Monteyne T, Coopman R, Kishabongo AS, Himpe J, Lapauw B, Shadid $S$, et al. Analysis of protein glycation in human fingernail clippings with near-infrared (NIR) spectroscopy as an alternative technique for the diagnosis of diabetes mellitus. Clin Chem Lab Med 2018;56:1552-9.

4. Dingari NC, Horowitz GL, Kang JW, Dasari RR, Barman I. Raman spectroscopy provides a powerful diagnostic tool for accurate determination of albumin glycation. PLoS One 2012; 7:e32406

5. Glenn JV, Beattie JR, Barrett L, Frizzell N, Thorpe SR, Boulton ME, et al. Confocal Raman microscopy can quantify advanced glycation end product (AGE) modifications in Bruch's membrane leading to accurate, nondestructive prediction of ocular aging. FASEB J 2007;21:3542-52.

6. Guilbert M, Said G, Happillon T, Untereiner V, Garnotel R, Jeannesson P, et al. Probing non-enzymatic glycation of type I 
collagen: a novel approach using Raman and infrared biophotonic methods. Biochim Biophys Acta 2013;1830:3525-31.

7. Jaisson S, Larreta-Garde V, Bellon G, Hornebeck W, Garnotel R, Gillery P. Carbamylation differentially alters type I collagen sensitivity to various collagenases. Matrix Biol 2007;26: 190-6.

8. Prentice BM, Caprioli RM, Vuiblet V. Label-free molecular imaging of the kidney. Kidney Int 2017;92:580-98.

9. Barman I, Dingari NC, Kang JW, Horowitz GL, Dasari RR, Feld MS. Raman spectroscopy-based sensitive and specific detection of glycated hemoglobin. Anal Chem 2012;84:2474-82.

10. Coopman R, Van de Vyver T, Kishabongo AS, Katchunga P, Van Aken EH, Cikomola J, et al. Glycation in human fingernail clippings using ATR-FTIR spectrometry, a new marker for the diagnosis and monitoring of diabetes mellitus. Clin Biochem 2017;50:62-7.

11. Beisswenger PJ, Moore LL, Curphey TJ. Relationship between glycemic control and collagen-linked advanced glycosylation end products in type I diabetes. Diabetes Care 1993;16:689-94.

12. Monnier VM, Genuth S, Sell DR. The pecking order of skin Advanced Glycation Endproducts (AGEs) as long-term markers of glycemic damage and risk factors for micro- and subclinical macrovascular disease progression in Type 1 diabetes. Glycoconj J 2016;33:569-79.

13. Yoshida S, Yoshida M, Yamamoto M, Takeda J. Optical screening of diabetes mellitus using non-invasive Fourier-transform infrared spectroscopy technique for human lip. J Pharm Biomed Anal 2013;76:169-76.

14. Kishabongo AS, Katchunga P, Van Aken EH, Speeckaert R, Lagniau S, Coopman R, et al. Glycation of nail proteins: from basic biochemical findings to a representative marker for diabetic glycation-associated target organ damage. PLoS One 2015;10:e0120112.

15. Bakan E, Bakan N. Glycosylation of nail in diabetics: possible marker of long-term hyperglycemia. Clin Chim Acta 1985;147: $1-5$.

16. Farhan KM, Sastry TP, Mandal AB. Comparative study on secondary structural changes in diabetic and non-diabetic human finger nail specimen by using FTIR spectra. Clin Chim Acta 2011;412:386-9.
17. Kishabongo AS, Katchunga P, Van Aken EH, Speeckaert MM, Lagniau S, Husein D, et al. Glycated nail proteins: a new approach for detecting diabetes in developing countries. Trop Med Int Health 2014;19:58-64.

18. Meerwaldt R, Links T, Graaff R, Thorpe SR, Baynes JW, Hartog J, et al. Simple noninvasive measurement of skin autofluorescence. Ann N Y Acad Sci 2005;1043:290-8.

19. Fokkens BT, Smit AJ. Skin fluorescence as a clinical tool for non-invasive assessment of advanced glycation and long-term complications of diabetes. Glycoconj J 2016;33:527-35.

20. Meerwaldt R, Hartog JW, Graaff R, Huisman RJ, Links TP, den Hollander NC, et al. Skin autofluorescence, a measure of cumulative metabolic stress and advanced glycation end products, predicts mortality in hemodialysis patients. J Am Soc Nephrol 2005;16:3687-93.

21. Jaisson S, Souchon PF, Desmons A, Salmon AS, Delemer B, Gillery P. Early formation of serum advanced glycation end-products in children with type 1 diabetes mellitus: relationship with glycemic control. J Pediatr 2016;172:56-62.

22. Lapolla A, Reitano R, Baccarin L, Sartore G, Plebani M, Fedele D. Pentosidine plasma levels and relation with metabolic control in diabetic patients. Horm Metab Res 2005;37:252-6.

23. Loomis SJ, Chen Y, Sacks DB, Christenson ES, Christenson RH, Rebholz CM, et al. Cross-sectional analysis of AGE-CML, SRAGE, and esRAGE with diabetes and cardiometabolic risk factors in a community-based cohort. Clin Chem 2017;63:980-9.

24. Jaisson S, Gillery P. Evaluation of nonenzymatic posttranslational modification-derived products as biomarkers of molecular aging of proteins. Clin Chem 2010;56:1401-12.

Corresponding author: Pr. Philippe Gillery, Service de BiochimiePharmacologie-Toxicologie, Pôle de Biologie Médicale et Pathologie, Hôpital Maison Blanche - CHU de Reims, 45 Rue Cognacq-Jay, F-51095 Reims, France, Phone: +33.3.26.78.39.52,

Fax: +33.3.26.78.38.82, E-mail: pgillery@chu-reims.fr; and Department of Biochemistry-Pharmacology-Toxicology, University Hospital of Reims, Reims, France

Stéphane Jaisson: Department of Biochemistry-PharmacologyToxicology, University Hospital of Reims, Reims, France 